\title{
PCL Cyst: A Rare Entity
}

\author{
Ravi Gupta ${ }^{1}$, Nitin Bither ${ }^{2 *}$ and Jagdeep Singh Atwal ${ }^{3}$
}

${ }^{1}$ Professor of Orthopedics, GMCH, India

${ }^{2}$ Shoulder and knee consultant Bither hospital, India

${ }^{3}$ Assistant Professor, GGS medical college, India

*Corresponding author: Nitin Bither, MS, DNB Shoulder and knee consultant Bither hospital, Sirhind, India

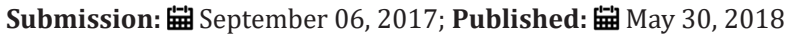

\begin{abstract}
Introduction: Intra-articular cysts and ganglia have been reported to be $0.6 \%$ and $2.0 \%$ in symptomatic knees. The low incidence causes lack of awareness resulting into missing of the diagnosis leading to events which otherwise are preventable.

Case presentation: We report a case of a 26 year old state level track and field athlete who presented with intermittent pain and sudden jerky movement of the right knee for the last 5 months who had consulted two specialists before coming to us and had also undergone two MRI examinations without any definite diagnosis. Diagnostic arthroscopy revealed PCL cyst with full thickness chondral defect on the medial condyle of the femur. Retrospectively, a close look at the preoperative MRI was able to pick up the PCL cyst which had been passed earlier as normal. The delay of 5 months in diagnosis and another 6 months for recuperation resulted in ending the career of the athlete.
\end{abstract}

Conclusion: In conclusion, reminder of this rare, though known pathology, is likely to facilitate an early diagnosis and treatment resulting into saving the career of the players.

Keywords: Posterior cruciate ligament; Cysts; Ganglion; Athlete; Knee

\section{Introduction}

Intraarticular cysts in the knee are rare and are reported to originate from cruciate ligaments, menisci, and the popliteus tendon [1]. These cysts may result into missed/delayed diagnosis due to nonspecific clinical symptoms and lack of awareness of the Case Report

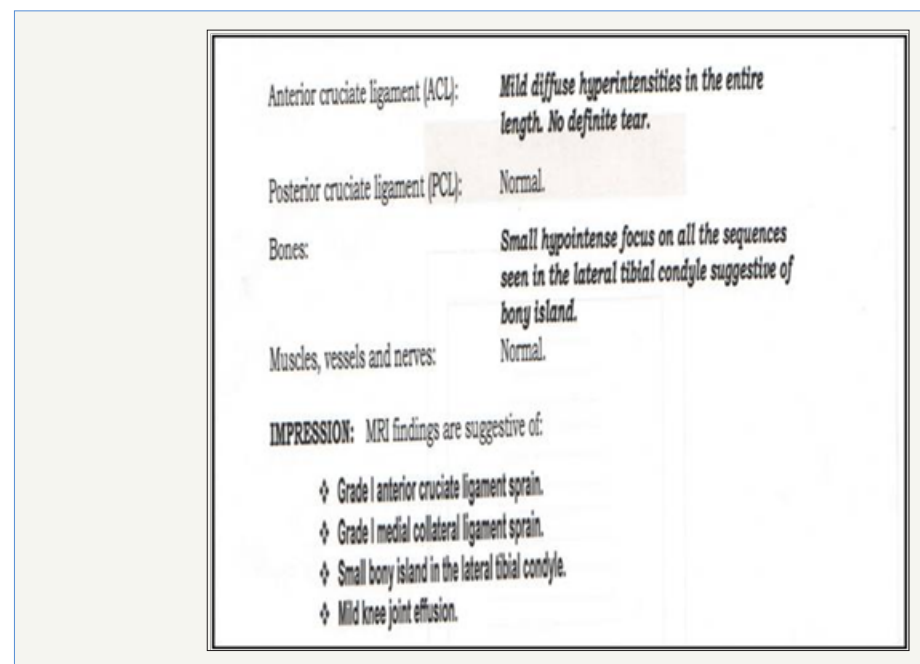

Figure 1: Reporting of Mri showing missed Pcl cyst. physician. We report a case of a posterior cruciate ligament (PCL) cyst which was missed clinically as well as radiologically for five months resulting into chondral damage to the articular cartilage and finally ending the career of the player.

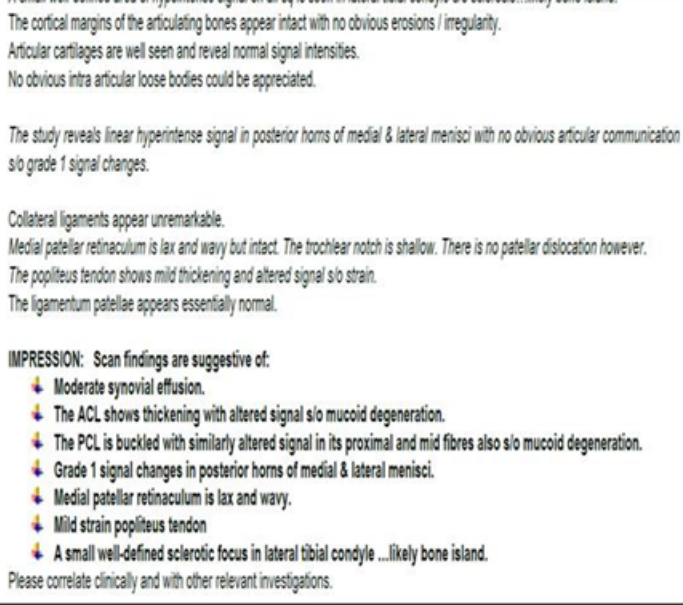


A 26 year old state level track and field female athlete presented with intermittent pain and sudden jerky movement of the right knee for the last 5 months. On specific questioning she denied the sensation of locking and explained that she felt a sudden thud and pain during the movement of terminal extension as if something slipped inside the knee especially during terminal extension of the knee and while shifting from swing to stance phase of the gait. After the episode of thud/jerk, her knee felt normal without any swelling or pain. It was so frequent that she felt disabled in activities of daily living. She had received two specialist opinion and treatment before presenting to us. Her x_ray and Magnetic resonance imaging (MRI) done twice during this period of 5 months had been reported as normal (Figure 1). She had also undergone ten sessions of physiotherapy during this period to the knee without any relief.

In the outpatient clinic, the examination of the knee including integrity of the ligaments and the tests for menisci were normal, but the patient was able to demonstrate the jerky thud in the terminal movement of extension of the knee which she was able to reproduce at least once when she extended the knee from flexion to extension 3-4 times.
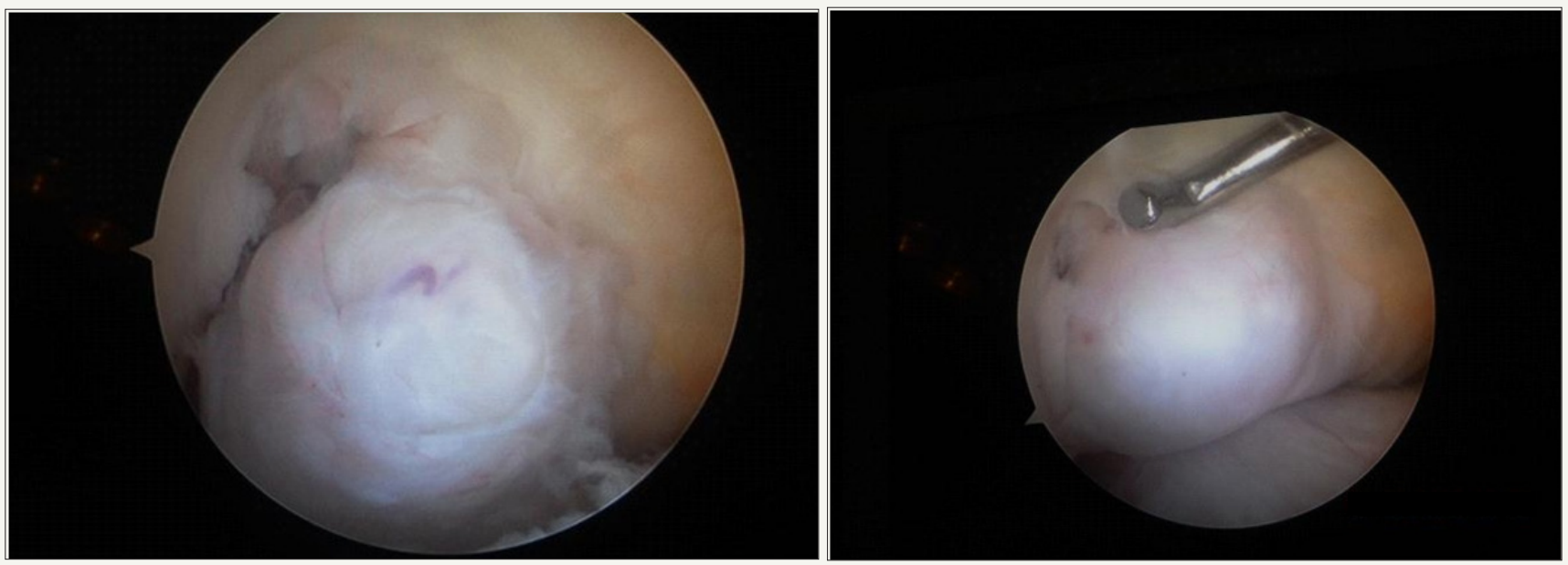

Figure 2: Arthroscopic view of Pcl Harbouring the cyst.

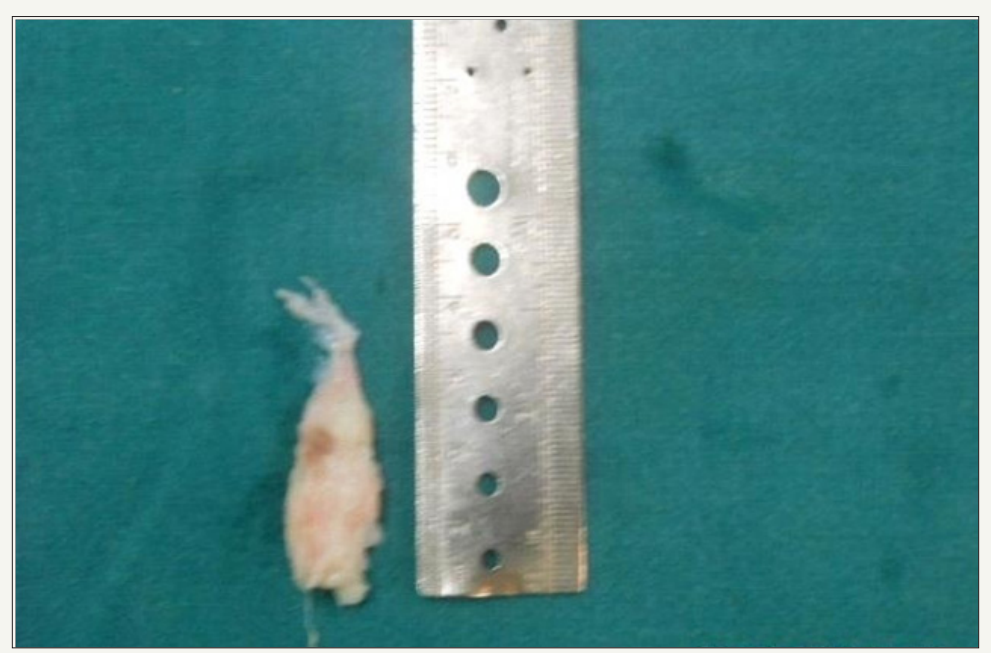

Figure 3: Pcl cyst after excision.

Patient was taken up for diagnostic arthroscopy. Just on the entry of the scope, the intercondylar notch of the femur was seen to be harboring a cyst floating freely which was attached to the PCL (Figure 2). We were able to excise the cyst retaining the integrity of its walls (without rupturing) by severing its attachment from the PCL (Figure 3 \& 4).

After the excision of the cyst, the routine examination of the knee showed a $1 \mathrm{~cm} \times 2 \mathrm{~cm}$ full thickness chondral defect on the medial condyle of the femur which was debrided to healthy hyaline cartilage and micro fracture was done (Figure 5). The histopathology of the excised cyst confirmed it to be a cyst lined by synovial cells. The patient was kept non/ partial weight bearing for 8 weeks to allow the healing of the micro fractures. At 6 months follow up patient had full function of the knee joint with Lysholm knee score of 100 . However, the patient could not go back to her place in the game due to long break from active sports. 


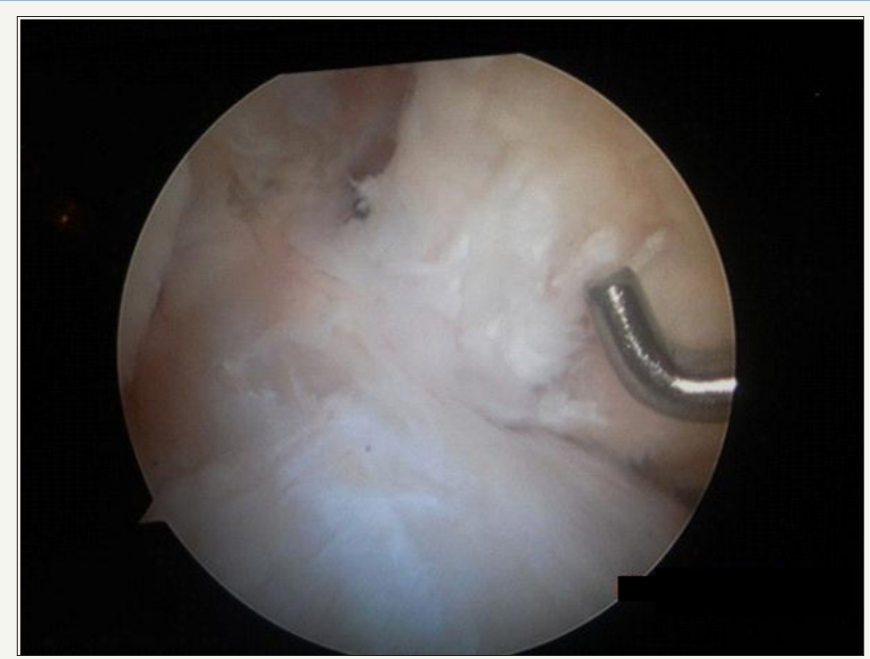

Figure 4: Intact Pcl post excision of cyst.
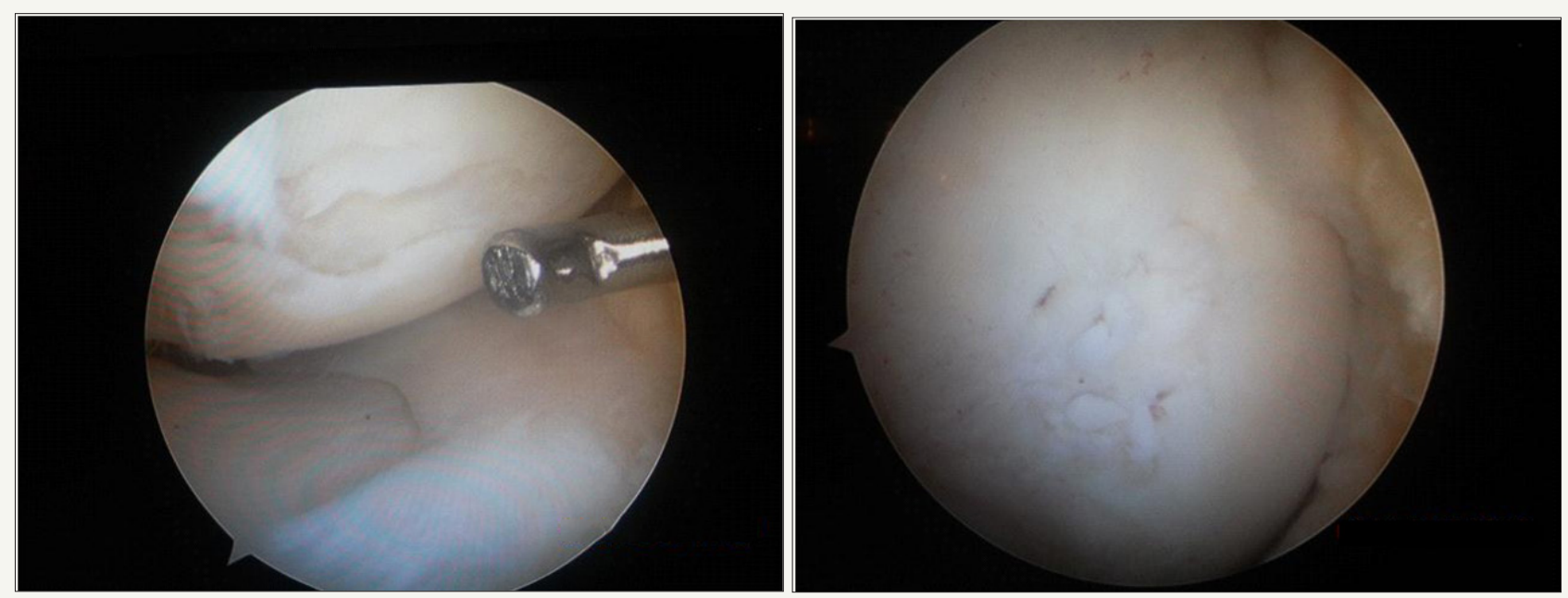

Figure 5: Medial chondral defect and after microfracture of the defect.

\section{Discussion}
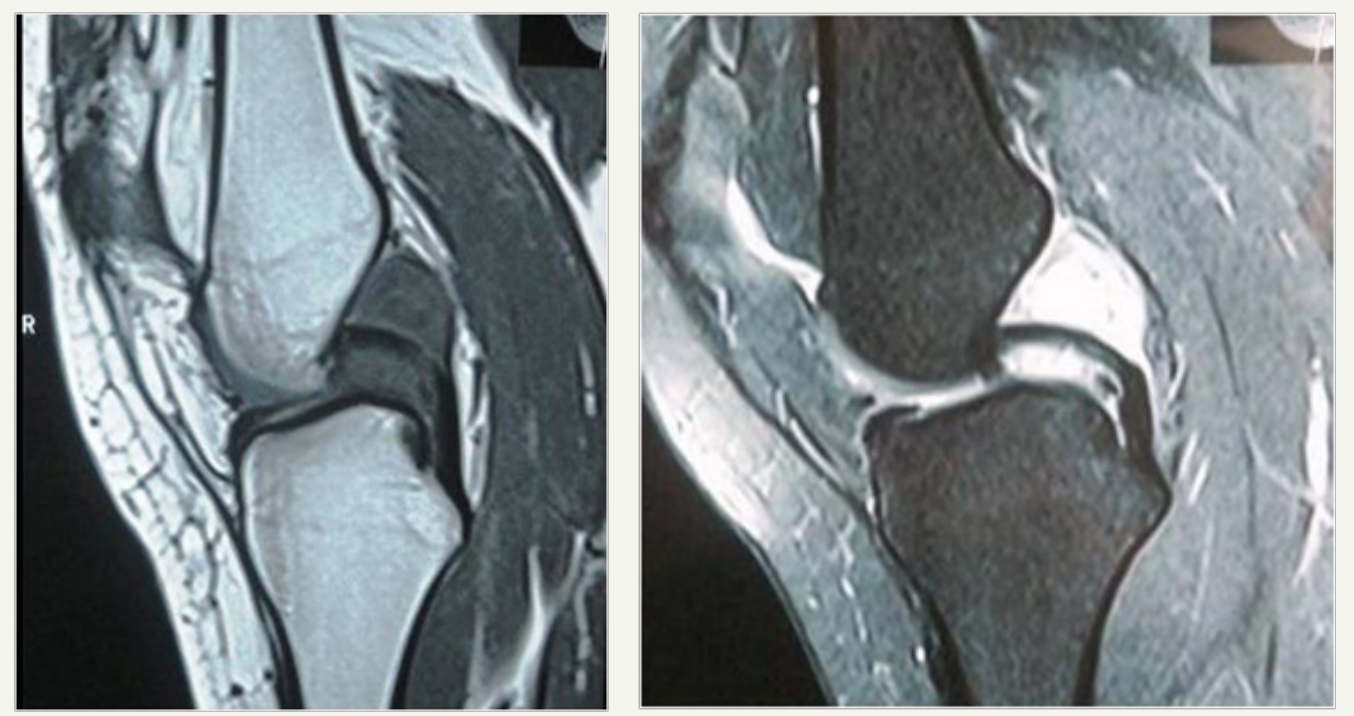

Figure 6: T1 and T2 weighted image showing a cyst (hypointense on T1 and hyperintense on T2) in the intercondylar notch. 
The low incidence of a clinical condition causes lack of awareness resulting into missing of the diagnosis because the treating physician as well as the radiologist focuses his/ her attention on the conditions which have been seen/ known by him. The dictum of eyes only sees what the mind knows is very well exemplified in the present case [2]. Neither the initial treating physicians nor the radiologists including the authors were able to pick up the diagnosis preoperatively because probably it never struck their mind. Retrospectively, when we had a relook at the MRI with the diagnosis established arthroscopically in our mind, we were able to see a space occupying lesion lying in the intercondylar notch (Figure 6).

The incidence of intra-articular cysts and ganglia has been reported to be $0.6 \%$ and $2.0 \%$ in arthroscopies performed on symptomatic knees $[3,4]$. The etiopathogenesis is still unclear and synovial tissue herniation, connective tissue degeneration after trauma being advocated as playing a role in the pathogenesis of these intra articular cysts [5]. Nearly two thirds of all ganglion cysts originate from the anterior cruciate ligament (ACL) [6]. Ganglion cysts associated with the ACL usually have a fusiform appearance and may be interspersed within the ACL fibers and usually arise from tibial insertion, while those associated with the PCL usually appear as a well-defined uniloculated or, more commonly, multiloculated cyst. PCL ganglion cysts tend to be located at the femoral or tibial insertions, rarely surround the ligament and do not communicate with a meniscal tear thus differentiating it from a pericruciate meniscal tear [7].

Although it can occur at any age, the peak incidence of intraarticular ganglion is approximately 20-40 years of age and occurs most commonly in males. Ganglion cysts arising from the PCL may limit flexion through mechanical block. Pain followed by stiffness is the most common presenting symptom without a preceding history of significant trauma but the patient may experience a spectrum of symptoms ranging from a sensation of fullness during knee motion, limitation in knee range of motion, and flexion or extension block. In some rare occasions there may be swelling, effusion, joint line tenderness, pain with McMurray's, and a clicking sensation thus posing a diagnostic challenge for the clinician [8-10]. In the present case, the patient had only one symptom of catching of the cyst during terminal extension of the knee joint; the clinical examination was unremarkable.

MR imaging is now considered the modality of choice for diagnosing cystic lesions of the knee. Ganglion cysts show usually homogeneous low signal intensity on T1- and high signal intensity on T2-weighted images [11]. Excellent results have been reported after removal of intra-articular cysts and ganglia; especially if the cyst was an isolated finding [6]. At the time of relook on the MRI in the present case, the presentation was quite typical. We believe that orthopedic surgeons should be well trained in the interpretation of the MRI pictures and not be misleading by the interpretation of the reporting personnel only. Furthermore, the need of a musculoskeletal radiologist is also well highlighted by the present report; both the MRIs in the present case had been reported by the general radiologist.

Our patient was totally free from symptoms at one year follow up and we do not expect the recurrence of symptoms. Unlike in other locations recurrence has not yet been a problem with arthroscopically treated ganglion cysts of the knee for unexplained reasons [12]. The presence of chondral defect in the medial condyle of the femur in the present case is most likely caused by the repeated episodes of catching of the cyst in the last 5 months. Since this was not able to be diagnosed and planned preoperatively for mosaicplasty, we resorted to micro fracture which was the readily available option in the operation room; we, otherwise, routinely perform mosaicplasty in such lesions in young patients.

\section{Conclusion}

In conclusion, a high index of suspicion is required in all the cases where the symptoms of the patients are not correlated with the clinical/radiological examination. Knowledge of even the rare pathologies is important to create awareness in the minds of the treating physicians so that the eyes can see, what is known by the mind.

\section{Consent}

This case report is being uploaded for submission with the consent of the patient.

\section{References}

1. Andrikoula IS, Vasiliadis SH, Tokis VA, Kosta P, Batistatou A, et al. (2007) Intra-articular ganglia of the knee joint associated with the anterior cruciate ligament: a report of 4 cases in 3 patients. Arthroscopy 23(7): $800 \mathrm{e} 1-800 \mathrm{e} 6$

2. Jairath UC, Spodick DH (2000) The eyes see only what the mind knows. J Emerg Med 19(3): 275-276.

3. Mansfield LT, Youngberg RA (1997) Intraarticular ganglia of the knee: prevalence, presentation, etiology, and management. AJR Am J Roentgenol 68(1): 123-127.

4. Parish EN, Dixon P, Cross MJ (2005) Ganglion cysts of the anterior cruciate ligament : a series of 15 cases. Arthroscopy 21(4): 445-447.

5. Soren (1966) Pathogenesis and treatment of ganglion. Clinical Orthopaedics and Related Research 48: 173-179.

6. Brown MF, Dandy DJ (1990) Intra-articular ganglia in the knee. Arthroscopy 6(4): 322-323.

7. Lektrakul N, Skaf A, Yeh L, Roger B, Schweitzer M, et al. (1999) Pericruciate meniscal cysts arising from tears of the posterior horn of the medial meniscus; MR imaging features that simulate posterior cruciate ganglion cysts. Am J Roentgenol 172(6): 1575-1579.

8. Shetty GM, Nha KW, Patil SP (2008) Ganglion cysts of the posterior cruciate ligament. The Knee 43: 419-424.

9. Seki K, Mine T, Tanaka H, Isida Y, Taguchi T (2006) Locked knee caused by intra-articular ganglion. Knee Surg Sports Traumatol Arthrosc 14(9): 859-861.

10. Tyrrell PM, Pullicino VN, McCall IW (2000) Intra-articular ganglion cysts of the cruciate ligament. Euro Radio 10: 1233-1238. 
11. Garcia A, Hodler J, Vaughn L, Haghighi P, Resnick D (1991) Intraarticular ganglion arising from the posterior cruciate ligament. Skeletal Radiol 20: 373-375.
12. Krudwig WK, Schulte KK, Heinemann C (2004) Intra-articular ganglion cysts of the knee joint: a report of 85 cases and review of the literature. Knee Surg Sports Traumatol Arthrosc 12(2): 123-129.

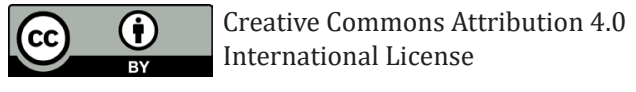

For possible submissions Click Here

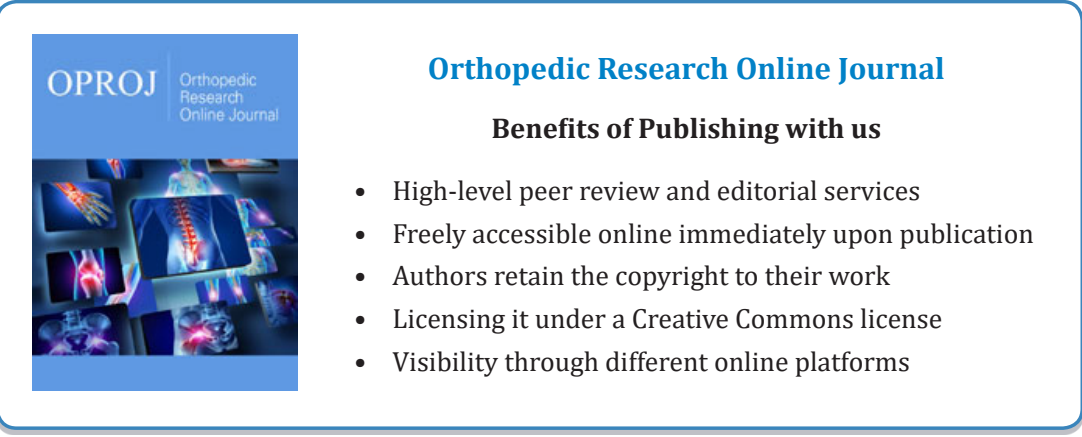

